\title{
Analytical Solutions of Fractional Walter's B Fluid with Applications
}

\author{
Qasem Al-Mdallal $\mathbb{D},{ }^{1}$ Kashif Ali Abro $\mathbb{D},{ }^{2}$ and Ilyas Khan ${ }^{3}$ \\ ${ }^{1}$ Department of Mathematical Sciences, UAE University, P.O. Box 15551, Al Ain, UAE \\ ${ }^{2}$ Department of Basic Sciences and Related Studies, Mehran University of Engineering Technology, Jamshoro, Pakistan \\ ${ }^{3}$ Basic Engineering Sciences Department, College of Engineering, Majmaah University, Al Majmaah, Saudi Arabia
}

Correspondence should be addressed to Qasem Al-Mdallal; q.almdallal@uaeu.ac.ae

Received 17 May 2017; Accepted 11 December 2017; Published 28 February 2018

Academic Editor: José Ángel Acosta

Copyright (C) 2018 Qasem Al-Mdallal et al. This is an open access article distributed under the Creative Commons Attribution License, which permits unrestricted use, distribution, and reproduction in any medium, provided the original work is properly cited.

\begin{abstract}
Fractional Walter's Liquid Model-B has been used in this work to study the combined analysis of heat and mass transfer together with magnetohydrodynamic (MHD) flow over a vertically oscillating plate embedded in a porous medium. A newly defined approach of Caputo-Fabrizio fractional derivative (CFFD) has been used in the mathematical formulation of the problem. By employing the dimensional analysis, the dimensional governing partial differential equations have been transformed into dimensionless form. The problem is solved analytically and solutions of mass concentration, temperature distribution, and velocity field are obtained in the presence and absence of porous and magnetic field impacts. The general solutions are expressed in the format of generalized Mittag-Leffler function $\mathbf{M}_{\Omega_{2}, \Omega_{3}}^{\Omega_{1}}(\chi)$ and Fox- $\mathbf{H}$ function $\mathbf{H}_{p, q+1}^{1, p}$ satisfying imposed conditions on the problem. These solutions have combined effects of heat and mass transfer; this is due to free convections differences between mass concentration and temperature distribution. Graphical illustration is depicted in order to bring out the effects of various physical parameters on flow. From investigated general solutions, the well-known previously published results in the literature have been recovered. Graphs are plotted and discussed for rheological parameters.
\end{abstract}

\section{Introduction}

The liquids related to non-Newtonian behavior have diverted the attention of various researchers, due to the involvement of non-Newtonian fluids in industrial processes and engineering. The prominent non-Newtonian liquids are liquid detergents, polymers, shampoos, cosmetic products, printer inks, blood at low shear rate, paints, colloidal fluids, mud, ice cream, suspension fluids, and several others. Due to diverse rheological characteristics in non-Newtonians liquids, no constitutive relation is present in literature. The investigators and scientists have recommended many models of nonNewtonian liquids due to nonlinearity between the shear rate and shear stress, such as Sisko's model [1], Jeffery's model [2], Oldroyd-B fluid model [3], Burgers elasto-viscous fluid model [4], Maxwell fluid model [5], differential third- and second-grade models $[6,7]$, and several others. Other than those, Walter's Liquid Model-B is a non-Newtonian model commonly known as viscoelastic model suggested by Walter [8]. The complex flow behavior of many industrial liquids can accurately be simulated by this viscoelastic model. The elastic properties and extensional polymer's behavior are also handled by Walter's Liquid Model-B, even it generates extremely nonlinear equations. Walter's Liquid Model-B problems using classical derivatives approach have been solved in numerous studies. However, Walter's Liquid Model-B problem with combined analysis of heat and mass transfer using fractional derivatives approach has not been investigated.

Factional calculus is the subject of science of differentiation and it was originated in 1695, while L'Hospital questioned Leibniz what would be the explanation of $d^{1 / 2} / d x^{1 / 2}$. This question led to a fruitful journey in the area of fractional calculus to study various fractional operators in which definitions of fractional operator along with significant properties have been studied by several mathematicians and scientists, for instance, Riemann-Liouville fractional derivative, 
Caputo fractional derivative, Caputo-Erdelyi-Kober fractional derivative, Caputo-Hadamard fractional derivative, and Caputo-Fabrizio fractional derivatives [9-18]. Due to distinct kernel representations in distinct function spaces generate diversity of definitions for fractional derivatives. These types of derivatives reveal few complications in applications; for instance, the Laplace transform of Riemann-Liouville derivative consists of terms without physical signification and its constant is not zero. These difficulties were eradicated by Caputo fractional derivative but they involve singular kernel. In order to avoid singularities, Caputo and Fabrizio have presented new fractional derivative, namely, Caputo-Fabrizio fractional derivatives based on exponential kernel $[9,11]$. Based on the exponential kernel with no singularity, this article is proposed to analyze the viscoelastic Walter's Liquid Model-B [19]. Atangana and Alqahtani [20] have analyzed the groundwater pollution by employing Caputo-Fabrizio fractional derivatives with numerical approximation. Alkahtani and Atangana [21] investigated the effect of Caputo-Fabrizio fractional derivatives on the surface of shallow water for controlling the wave movement. Hristov [22] has traced out the analytical solution for steady-state heat conduction using Caputo-Fabrizio space-fractional derivative with nonsingular fading memory. Atangana and Baleanu [23] presented a heat transfer model by implementing Caputo-Fabrizio space-fractional derivative. Nadeem et al. [24] observed the effects of two types of fractional derivatives, namely, CaputoFabrizio (CF) fractional operator and Atangana-Baleanu (AB) fractional operator for free convection flow of generalized Casson fluid. They suggested that the velocities obtained via Atangana-Baleanu ( $\mathrm{AB}$ ) fractional operator and CaputoFabrizio (CF) fractional operator are identical. Nadeem et al. [25] investigated MHD flow of a second-grade fluid via Caputo-Fabrizio derivatives of fractional order by invoking integral transform over an oscillating boundary. Kashif and Muhammad [26] observed heat transfer analysis for the flow of a second-grade fluid using Caputo-Fabrizio derivatives in which they presented analytic solutions using Mittag-Leffler function and Fox- $H$ function. Nadeem et al. [27] presented a comparative study for generalized Casson fluid by implementing Atangana-Baleanu (AB) fractional operator and Caputo-Fabrizio (CF) fractional operator. For the sake of simplicity, we include here few very recent attempts on AtanganaBaleanu (AB) fractional operator and Caputo-Fabrizio (CF) fractional operator as well [28-30]. In brevity, some recent studies regarding fractional derivatives can be found in [11, 31-34]. Our aim is to analyze MHD flow of fractionalized Walter's Liquid Model-B with heat and mass transfer in porous medium analytically using newly defined approach of Caputo-Fabrizio fractional derivative. By employing dimensional analysis, the dimensional governing partial differential equations have been reduced in terms of dimensionless form. The analytical investigation is performed for the solutions of mass concentration, temperature distribution, and velocity profile in presence and absence of porous and magnetic field impacts. The general solutions are expressed in the format of generalized Mittag-Leffler function $\mathbf{M}_{\Omega_{2}, \Omega_{3}}^{\Omega_{1}}(\chi)$ and Fox-H function $\mathbf{H}_{p, q+1}^{1, p}$ satisfying imposed conditions on the problem. These solutions have combined effects of heat and mass transfer; this is due to free convections differences between mass concentration and temperature distribution. Graphical illustration is depicted in order to bring out the effects of various physical parameters on flow.

\section{Preliminaries}

In this section, we present some essential information about the Caputo-Fabrizio fractional derivative that will be used in this paper. Firstly, we introduce the definition of the CaputoFabrizio fractional derivative of order $\psi$.

Definition 1. The Caputo-Fabrizio fractional derivative of order $\psi \in(0,1]$ is defined as in the following [9-11]:

$$
\frac{\partial^{\psi}}{\partial t^{\psi}} p(t)=\int \frac{p^{\prime}(\delta)}{(1-\psi)} \exp \left(\frac{-(t-\delta) \psi}{(1-\psi)}\right) d \delta
$$

where the fractional operator $\partial^{\psi} / \partial t^{\psi}$ is the so-called CaputoFabrizio fractional operator. It is well-known that the Laplace transform of the Caputo-Fabrizio fractional derivative is given by

$$
L\left[\frac{\partial^{\psi} p(t)}{\partial t^{\psi}}\right]=\frac{\eta L\{p(\eta)\}-p(0)}{\eta(1-\psi)+\psi} .
$$

On the other hand, it essential to note that Caputo-Fabrizio fractional operator can be extended significantly by letting $\psi=1$ in (2); we arrive at

$$
\begin{aligned}
\lim _{\psi \rightarrow 1} L\left[\frac{\partial^{\psi} p(t)}{\partial t^{\psi}}\right] & =\lim _{\psi \rightarrow 1}\left[\frac{\eta L\{p(\eta)\}-p(0)}{\eta(1-\psi)+\psi}\right] \\
& =\eta L\{p(\eta)\}-p(0)=L\left\{p^{\prime}(\eta)\right\}
\end{aligned}
$$

\section{Governing Equations}

The constitutive equations govern the flow of Walters'-B fluid are

$$
\begin{gathered}
\operatorname{div} \overrightarrow{\mathbf{V}}=0, \\
\rho \frac{d \overrightarrow{\mathbf{V}}}{d t}+\operatorname{grad} p-\rho g-\operatorname{div} \mathbf{T}, \\
\mathbf{T}=k_{1} \mathbf{A}_{1}^{2}-k_{0} \mathbf{A}_{2}+\mu \mathbf{A}_{1}-p \mathbf{I},
\end{gathered}
$$

where $\mathbf{T}, k_{1}, k_{0}, \mathbf{A}_{1}, \mathbf{A}_{2}, \mu, \mathbf{I}, p$ are Cauchy stress tensor, cross viscosity and viscosity of the fluid, kinematic tensors, coefficient of viscosity, identity tensor, and scalar part of the pressure, respectively. In addition, $\mathbf{A}_{2}$ is defined as follows:

$$
\begin{aligned}
& \mathbf{A}_{2}=\frac{d \mathbf{A}_{1}}{d t}+\mathbf{A}_{1}(\operatorname{grad} \overrightarrow{\mathbf{V}})^{T}+\mathbf{A}_{1}(\operatorname{grad} \overrightarrow{\mathbf{V}}) \\
& \mathbf{A}_{1}=(\operatorname{grad} \overrightarrow{\mathbf{V}})^{T}+(\operatorname{grad} \overrightarrow{\mathbf{V}}),
\end{aligned}
$$

where $d \mathbf{A}_{1} / d t$ is the material time derivative and $\overrightarrow{\mathbf{V}}$ is the velocity vector. According to the condition of Walters'-B fluid 
as $k_{0}<0, k_{1}, \mu>0, \mathbf{A}_{1}$ is taken same for generalized Walters'$B$ fluid and $\mathbf{A}_{2}$ takes place as

$$
\mathbf{A}_{2}=\mathbf{A}_{1}(\operatorname{grad} \overrightarrow{\mathbf{V}})^{T}+\mathbf{A}_{1}(\operatorname{grad} \overrightarrow{\mathbf{V}})+\frac{\partial^{\psi}}{\partial t^{\psi}} \mathbf{A}_{1}
$$

$$
0<\psi<1
$$

where the fractional operator $\partial^{\psi} / \partial t^{\psi}$ is the so-called CaputoFabrizio fractional operator of order $0<\psi<1$ as previously published papers in open literature $[9,11]$ defined as

$$
\frac{\partial^{\psi}}{\partial t^{\psi}} p(t)=\int \frac{p^{\prime}(t)}{(1-\psi)} \exp \left(\frac{-(t-\delta) \psi}{(1-\psi)}\right) d \delta
$$

$$
0<\psi<1 \text {. }
$$

For the problem with above assumption, the velocity field for oscillating flow is assumed as follows:

$$
\overrightarrow{\mathbf{V}}=(u(y, t), 0,0)
$$

In $(8), u(y, t)$ is the velocity field in the $x$ direction and (4a) and (4b) are identically fulfilled due to constrain of the incompressibility and balance of the linear momentum, respectively, while without external pressure gradient in flow produces the partial differential equations for the Walters'-B fluid as follows [19]:

$$
\begin{aligned}
\lambda^{\psi} \frac{\partial^{\psi} u^{*}\left(y^{*}, t^{*}\right)}{\partial t^{\psi^{*}}}= & \nu \lambda \frac{\partial^{2} u^{*}\left(y^{*}, t^{*}\right)}{\partial y^{*^{2}}} \\
& -\frac{\lambda^{\psi} k_{0}}{\rho} \frac{\partial^{\psi}}{\partial t^{\psi^{*}}} \frac{\partial^{2} u^{*}\left(y^{*}, t^{*}\right)}{\partial^{2} y^{*}}+\vec{g} \rho .
\end{aligned}
$$

\section{Mathematical Model of Fractional Walter's-B Liquid}

Assume an unsteady electrically conducting incompressible free convection porous flow of a Walters'-B fluid over an oscillating plate. As described in the geometry Figure 1, the $y$-axis is normal to the plate and the $x$-axis is taken parallel to the plate. In the start, the plate and fluid both are at rest at uniform concentration $C_{\infty}$ and temperature $T_{\infty}$. At the time $t=0^{+}$, concentration and temperature rise up to $C_{w}$ and $T_{w}$, respectively. For such fluid motion, the governing partial differential equations for velocity profile, mass concentration, and temperature distribution are as follows:

$$
\begin{aligned}
\lambda^{\psi} \frac{\partial^{\psi} u(y, t)}{\partial t^{\psi}}= & \nu \lambda \frac{\partial^{2} u(y, t)}{\partial y^{2}}-\frac{\lambda^{\psi} k_{0}}{\rho} \frac{\partial^{\psi}}{\partial t^{\psi}} \frac{\partial^{2} u(y, t)}{\partial^{2} y} \\
& +\left(C-C_{\infty}\right) \beta_{C} \lambda g \\
& +\left(T-T_{\infty}\right) \beta_{T} \lambda g
\end{aligned}
$$

$$
\begin{array}{r}
-\left(\frac{\Phi \lambda}{\rho}+\frac{B_{0}^{2} \sigma \lambda}{\rho}\right) u(y, t), \\
\lambda^{\psi} \frac{\partial^{\psi} C(y, t)}{\partial t^{\psi}}=D \lambda \frac{\partial^{2} C(y, t)}{\partial y^{2}}, \\
\lambda^{\psi} \frac{\partial^{\psi} T(y, t)}{\partial t^{\psi}}=\frac{\lambda k}{c_{p} \rho} \frac{\partial^{2} T(y, t)}{\partial y^{2}},
\end{array}
$$

corresponding to initial and boundary conditions as

$$
\begin{aligned}
u(y, 0) & =0, \\
C(y, 0) & =C_{\infty}, \\
T(y, 0) & =T_{\infty}, \\
t & \leq 0, \\
y & >0, \\
u(0, t) & =U H(t) \cos (\omega t), \\
C(0, t) & =C_{w}, \\
T(0, t) & =T_{w}, \\
t & >0, \\
u(\infty, t) & =0, \\
C(\infty, t) & =C_{\infty}, \\
T(\infty, t) & =T_{\infty} .
\end{aligned}
$$

Here, $k_{0}, \rho, u(y, t), v, \beta_{C}, \beta_{T}, \Phi, g, k, D, c_{p}, C(y, t), T(y, t)$ are the Walters'-B viscoelasticity parameter, fluid density, velocity of fluid, the kinematic viscosity, the volumetric mass coefficient of expansion, the volumetric thermal coefficient of expansion, porosity, the gravitational acceleration, the thermal conductivity of the fluid, the mass diffusivity, the specific heat of the fluid at constant pressure, the species concentration, and the fluid temperature, respectively.

Meanwhile, we introduce the nondimensional parameters in (10)-(11) in the following manners:

$$
\begin{aligned}
u^{*} & =\frac{u}{U_{0}}, \\
y^{*} & =\frac{U_{0} y}{v}, \\
t^{*} & =\frac{t}{\lambda}, \\
C & =\frac{C-C_{\infty}}{C_{w}-C_{\infty}}, \\
T & =\frac{T-T_{\infty}}{T_{w}-T_{\infty}} .
\end{aligned}
$$




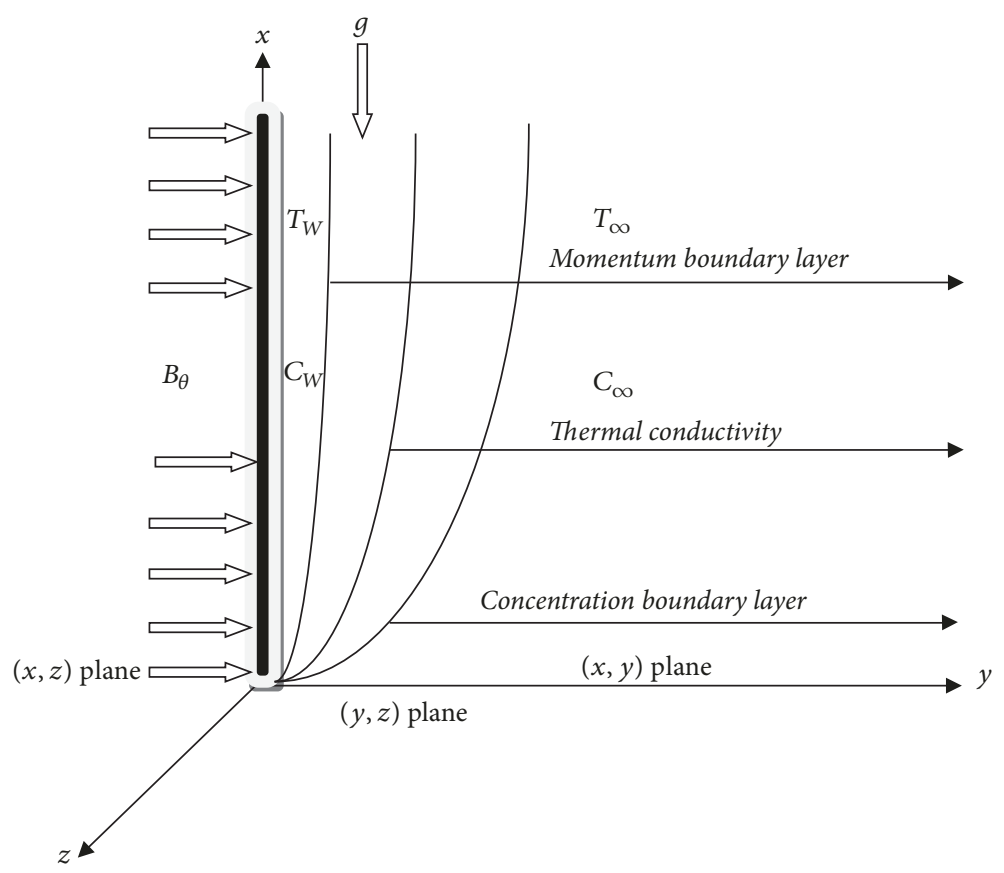

FIGURE 1: Geometry of the model.

Omitting * symbol for simplicity, we arrive at governing equations and imposed conditions as follows:

$$
\begin{aligned}
\frac{\partial^{\psi} u(y, t)}{\partial t^{\psi}}= & \left(R_{e}-\Gamma \frac{\partial^{\psi}}{\partial t^{\psi}}\right) \frac{\partial^{2} u(y, t)}{\partial y^{2}}-\Phi u(y, t) \\
& -M u(y, t)+G_{r} T(y, t) \\
& +G_{m} C(y, t) \\
\operatorname{Sc}_{\text {eff }} \frac{\partial^{\psi} C(y, t)}{\partial t^{\psi}}= & \frac{\partial^{2} C(y, t)}{\partial y^{2}}, \\
\operatorname{Pr}_{\mathrm{eff}} \frac{\partial^{\psi} T(y, t)}{\partial t^{\psi}}= & \frac{\partial^{2} T(y, t)}{\partial y^{2}}
\end{aligned}
$$

with initial and boundary conditions:

$$
\begin{aligned}
& u(y, 0)=C(y, 0)=T(y, 0)=0, \\
& u(0, t)=U H(t) \cos (\omega t), \\
& C(0, t)=T(0, t)=1, \\
& u(\infty, t)=C(\infty, t)=T(\infty, t)=0 .
\end{aligned}
$$

Here, Reynold number is $\operatorname{Re}=U^{2} \lambda / \nu$, Schmidt number is Sc $=\nu / D$, Prandtl number is $\operatorname{Pr}=c_{p} \mu / k$, thermal Grashof number is $\mathrm{Gr}=\left(T_{w}-T_{\infty}\right) \beta_{T} \lambda g / U_{0}$, Mass Grashof number is $\mathrm{Gr}=\left(C_{w}-C_{\infty}\right) \beta_{C} \lambda g / U_{0}$, effective Schmidt number is $\mathrm{Sc}_{\text {eff }}=$ $\mathrm{Re} / \mathrm{Sc}$, effective Prandtl number is $\mathrm{Pr}_{\text {eff }}=\mathrm{Re} / \mathrm{Pr}$, porosity is $\Phi=\lambda \varphi / K$, and $\Gamma=U_{0}^{2} k_{0} / \nu^{2} \rho$.

\section{Solution of Problem}

5.1. Analytic Solution of Temperature and Concentration Distribution. Employing Laplace transform on (14) and (15) along with initial and boundary conditions (16) and by substituting $\xi=1 /(1-\psi)$, we find that

$$
\begin{aligned}
& \bar{C}(y, \eta)=\frac{1}{\eta} \exp \left(-y \sqrt{\frac{\mathrm{Sc}_{\mathrm{eff}} \psi \eta}{\eta+\psi \xi}}\right), \\
& \bar{T}(y, \eta)=\frac{1}{\eta} \exp \left(-y \sqrt{\frac{\operatorname{Pr}_{\mathrm{eff}} \psi \eta}{\eta+\psi \xi}}\right) .
\end{aligned}
$$

Expanding (17) in terms of series form, we arrive at

$$
\begin{aligned}
\bar{C}(y, \eta) \\
=\frac{1}{\eta} \\
\quad+\sum_{p=1}^{\infty} \frac{\left(-y \sqrt{\psi \mathrm{Sc}_{\mathrm{eff}}}\right)^{p}}{p !} \sum_{q=0}^{\infty} \frac{(-\psi \xi)^{q} \Gamma(q+p / 2)}{q ! \Gamma(p / 2)} \frac{1}{\eta^{q+1}},
\end{aligned}
$$




$$
\begin{aligned}
\bar{T}(y, \eta) \\
=\frac{1}{\eta} \\
\quad+\sum_{p=1}^{\infty} \frac{\left(-y \sqrt{\psi \operatorname{Pr}_{\text {eff }}}\right)^{p}}{p !} \sum_{q=0}^{\infty} \frac{(-\psi \xi)^{q} \Gamma(q+p / 2)}{q ! \Gamma(p / 2)} \frac{1}{\eta^{q+1}} .
\end{aligned}
$$

Apply inverse Laplace transform on (18) and express mass concentration and temperature distribution in terms of generalized Mittag-Leffler function as

$$
\begin{aligned}
& C(y, t)=1+\sum_{p=1}^{\infty} \frac{\left(-y \sqrt{\psi \mathrm{Sc}_{\mathrm{eff}}}\right)^{p}}{p !} \mathbf{M}_{1,1}^{p / 2}(-\xi \psi t), \\
& T(y, t)=1+\sum_{p=1}^{\infty} \frac{\left(-y \sqrt{\psi \mathrm{Pr}_{\mathrm{eff}}}\right)^{p}}{p !} \mathbf{M}_{1,1}^{p / 2}(-\xi \psi t) .
\end{aligned}
$$

Here, the property of Mittag-Leffler function is defined as follows [33]:

$$
\begin{aligned}
\mathbf{M}_{\Omega_{2}, \Omega_{3}}^{\Omega_{1}}(\chi) & =t^{\Omega_{3}-1} \mathbf{E}_{\Omega_{2}, \Omega_{3}}^{\Omega_{1}}(\chi) \\
& =\sum_{f}^{\infty} \frac{(\chi)^{f} \Gamma\left(\Omega_{1}+f\right) t^{\Omega_{3}-1}}{f ! \Gamma\left(\Omega_{1}\right) \Gamma\left(\Omega_{3}+\Omega_{2} f\right)}, \\
\operatorname{Re}\left(\Omega_{2}\right) & >0, \\
\operatorname{Re}\left(\Omega_{3}\right) & >0 .
\end{aligned}
$$

5.2. Analytic Solution of Velocity Field. Employing Laplace transform on (14) along with initial and boundary conditions (16) and by substituting $\xi=1 /(1-\psi)$, we find that

$$
\begin{gathered}
\frac{\partial^{2} \bar{w}(y, \eta)}{\partial y^{2}}-\left(\frac{(M+\phi)(\eta+\xi)+\psi \eta}{\operatorname{Re}(\eta+\psi \xi)-\psi \xi \Gamma}\right) \bar{w}(y, \eta) \\
+\frac{(\eta+\psi \xi)\left\{G_{m} \bar{C}(y, \eta)+G_{r} \bar{T}(y, \eta)\right\}}{\operatorname{Re}(\eta+\psi \xi)-\psi \xi \Gamma}=0,
\end{gathered}
$$

and, by simplifying (22) and using imposed conditions, we get

$$
\begin{aligned}
& w(y, \eta)=\frac{U \eta}{\eta^{2}+\omega^{2}} \\
& \cdot \exp \left(-y \sqrt{\frac{\psi \eta+(M+\phi)(\eta+\psi \xi)}{R_{e}(\eta+\psi \xi)-\psi \xi \Gamma}}\right)-\frac{G_{m}}{R_{e}^{2}} \frac{1}{\eta}
\end{aligned}
$$

$$
\begin{aligned}
& \cdot \exp \left(-y \sqrt{\frac{\mathrm{Sc}_{\mathrm{eff}} \eta \psi}{\eta+\psi \xi}}\right) \frac{\left(\Lambda_{1} \eta^{2}+\Lambda_{2} \eta+\Lambda_{3}\right)}{\left(\eta^{2}+2 \Lambda_{4} \eta+\Lambda_{4}^{2}\right)}-\frac{G_{r}}{R_{e}} \\
& \cdot \frac{1}{\eta} \exp \left(-y \sqrt{\frac{\operatorname{Pr}_{\mathrm{eff}} \eta \psi}{\eta+\psi \xi}}\right) \frac{\left(\Lambda_{5} \eta^{2}+\Lambda_{6} \eta+\Lambda_{3}\right)}{\left(\eta^{2}+2 \Lambda_{4} \eta+\Lambda_{4}^{2}\right)}
\end{aligned}
$$

$$
\begin{aligned}
\Lambda_{1} & =\left(\operatorname{ReSc}_{\mathrm{eff}} \psi-\psi^{2}-M-\Phi\right), \\
\Lambda_{2} & =\left(\operatorname{ReSc}_{\mathrm{eff}} \psi^{2} \xi-\xi \psi^{2}-2 \psi M \xi-2 \Phi \xi \psi\right. \\
& \left.-\operatorname{Sc}_{\mathrm{eff}} \xi \psi^{2}\right), \\
\Lambda_{3} & =\left(-M \psi^{2} \xi^{2}-\Phi \psi^{2} \xi^{2}\right), \\
\Lambda_{4} & =\left(\psi \xi-\frac{\psi \xi \Gamma}{\operatorname{Re}}\right), \\
\Lambda_{5} & =\left(\operatorname{Re} \operatorname{Pr}_{\mathrm{eff}} \psi-\psi^{2}-M-\Phi\right), \\
\Lambda_{6} & =\left(\operatorname{Re}_{\operatorname{Pr}} \psi_{\mathrm{eff}}^{2} \xi-\xi \psi^{2}-2 \psi M \xi-2 \Phi \xi \psi\right. \\
& \left.-\operatorname{Pr}_{\mathrm{eff}} \xi \psi^{2}\right) .
\end{aligned}
$$

Expressing (23) in more suitable form, we obtain

$$
\begin{aligned}
& w(y, \eta)=\frac{U \eta}{\eta^{2}+\omega^{2}}+\frac{U \eta}{\eta^{2}+\omega^{2}} \\
& \cdot \sum_{p=1}^{\infty} \frac{1}{p !}\left(-y \sqrt{\frac{\psi+M+\Phi}{\operatorname{Re}}}\right)^{p} \\
& \cdot \sum_{q=0}^{\infty} \frac{1}{q !}\left(\frac{-\psi \xi M-\psi \xi \Phi}{\psi+M+\Phi}\right)^{q} \\
& \times \sum_{m=0}^{\infty} \frac{\left(\operatorname{Re} \psi \xi \Gamma-\operatorname{Re}^{2} \psi \xi\right)^{m} \Gamma(p / 2+1) \Gamma(p / 2+m)}{m ! \Gamma(p / 2) \Gamma(p / 2-q+1) \eta^{q-p-m}} \\
& -\frac{G_{m}}{R_{e}^{2}} \frac{1}{\eta} \exp \left(-y \sqrt{\frac{\operatorname{Sc}_{\mathrm{eff}} \eta \psi}{\eta+\psi \xi}}\right) \frac{\left(\eta-\eta_{1}\right)\left(\eta-\eta_{2}\right)}{\left(\eta-\eta_{3}\right)\left(\eta-\eta_{4}\right)} \\
& -\frac{G_{r}}{R_{e}} \frac{1}{\eta} \exp \left(-y \sqrt{\frac{\operatorname{Pr}_{\mathrm{eff}} \eta \psi}{\eta+\psi \xi}}\right) \frac{\left(\eta-\eta_{5}\right)\left(\eta-\eta_{6}\right)}{\left(\eta-\eta_{3}\right)\left(\eta-\eta_{4}\right)} \\
& \Lambda_{1} \eta^{2}+\Lambda_{2} \eta+\Lambda_{3}=\left(\eta-\eta_{1}\right)\left(\eta-\eta_{2}\right), \\
& \eta^{2}+2 \Lambda_{4} \eta+\Lambda_{4}^{2}=\left(\eta-\eta_{3}\right)\left(\eta-\eta_{4}\right),
\end{aligned}
$$


$\Lambda_{5} \eta^{2}+\Lambda_{6} \eta+\Lambda_{3}=\left(\eta-\eta_{5}\right)\left(\eta-\eta_{6}\right)$.
Applying inverse Laplace transform on (25) and expressing velocity field in terms of generalized special functions and Fox- $H$ function, we arrive at

$$
\begin{aligned}
& w(y, t)=U H(t) \cos \omega t+U H(t) \sum_{p=1}^{\infty} \frac{1}{p !}\left(-y \sqrt{\frac{\psi+M+\Phi}{\operatorname{Re}}}\right)^{p} \sum_{q=0}^{\infty} \frac{1}{q !}\left(\frac{-\psi \xi M-\psi \xi \Phi}{\psi+M+\Phi}\right)^{q} \\
& \cdot \int_{0}^{t} \cos \omega(t-\tau) \times \mathbf{H}_{2,4}^{1,2}\left[\left(\frac{t_{1}}{t}\right) \mid(0,1),\left(1-\frac{p}{2}, 0\right),\left(q-\frac{p}{2}, 0\right),(p-q+1,-1)\right] t^{q-p-1} d \tau-\frac{G_{m}}{R_{e}^{2}} \\
& \cdot \int_{0}^{t} \varphi\left(y, t, \psi \mathrm{Sc}_{\mathrm{eff}}, \psi \xi\right) \times\left[\frac{\left(\eta_{1} \eta_{2}-\eta_{1} \eta_{3}-\eta_{2} \eta_{3}+\eta_{3}^{2}\right)}{\left(\eta_{3}-\eta_{4}\right)} \exp \left\{\eta_{3}(t-\tau)\right\}-\frac{\left(\eta_{1} \eta_{2}-\eta_{1} \eta_{4}-\eta_{2} \eta_{4}+\eta_{4}^{2}\right)}{\left(\eta_{3}-\eta_{4}\right)} \exp \left\{\eta_{4}(t-\tau)\right\}\right] d \tau \\
& -\frac{G_{r}}{R_{e}}\left(1-\frac{p}{2},-1\right) \\
& \cdot \int_{0}^{t} \varphi\left(y, t, \psi \operatorname{Pr}_{\mathrm{eff}}, \psi \xi\right)\left[\frac{\left(\eta_{5} \eta_{6}-\eta_{5} \eta_{3}-\eta_{6} \eta_{3}+\eta_{3}^{2}\right)}{\left(\eta_{3}-\eta_{4}\right)} \exp \left\{\eta_{3}(t-\tau)\right\}-\frac{\left(\eta_{5} \eta_{6}-\eta_{5} \eta_{4}-\eta_{6} \eta_{4}+\eta_{4}^{2}\right)}{\left(\eta_{3}-\eta_{4}\right)} \exp \left\{\eta_{4}(t-\tau)\right\}\right] d \tau
\end{aligned}
$$

where $t_{1}=\operatorname{Re} \psi \xi \Gamma-\operatorname{Re}^{2} \psi \xi$ and the property of Fox-H function is as follows $[33,35]$ :

$$
\begin{aligned}
& \sum_{a}^{\infty} \frac{(-\Delta)^{a} \prod_{j=1}^{p} \Gamma\left(u_{j}+U_{j} a\right)}{a ! \prod_{j=1}^{q} \Gamma\left(v_{j}+V_{j} a\right)}=\mathbf{H}_{p, q+1}^{1, p}\left[\Delta \mid \begin{array}{c}
\left(1-u_{1}, U_{1}\right),\left(1-u_{2}, U_{2}\right),\left(1-u_{3}, U_{3}\right), \ldots,\left(1-u_{p}, U_{p}\right) \\
(0,1),\left(1-v_{1}, V_{1}\right),\left(1-v_{2}, V_{2}\right),\left(1-v_{3}, V_{3}\right), \ldots,\left(1-v_{q}, V_{q}\right)
\end{array}\right], \\
& L^{-1}\left\{\frac{1}{\eta} \exp \left(-y \sqrt{\frac{\mathrm{Sc}_{\mathrm{eff}} \eta \psi}{\eta+\psi \xi}}\right)\right\}=\varphi\left(y, t, \psi \mathrm{Sc}_{\mathrm{eff}}, \psi \xi\right) .
\end{aligned}
$$

Equations (19), (20), and (27) are the general solutions of mass concentration, temperature distribution, and velocity profile, respectively. From our general solutions, various solutions have recovered. When $\Phi=0$, the solutions are termed in the absence of porous effects, such solutions are obtained by Farhad et al. [16, see equation (28)]. Furthermore, setting $G_{m}=M=0$ and $\operatorname{Re}=1$ the solutions are explored in the absence of magnetic field and mass transfer; such solutions are quite identical obtained by Khan et al. [36]. Newtonian behavior of the solutions can also be achieved by employing $\Gamma=0$. It is worth pointing out that our analytical solutions are approached in terms of newly defined CaputoFabrizio fractionalized solutions and can be converted for ordinary differential operator by setting $\psi=1$.

\section{Numerical Results and Discussion}

The purpose of present analysis is to highlight the effects of fractionalized Walter's Liquid Model-B with heat and mass transfer in a porous medium analytically using newly defined approach of Caputo-Fabrizio fractional derivative. The analytical investigation is performed for the solutions of mass concentration, temperature distribution, and velocity profile in the presence and absence of porous medium and magnetic field effects. The general solutions are expressed in the form of generalized Mittag-Leffler function $\mathbf{M}_{\Omega_{2}, \Omega_{3}}^{\Omega_{1}}(\chi)$ and Fox-H function $\mathbf{H}_{p, q+1}^{1, p}$ satisfying imposed conditions on the problem. Figure 1 shows the physical model of the problem. The graphical illustration is depicted in order to bring out the effects of various physical parameters on flow as enumerated below in Figures 2-9.

(i) Figure 2 shows the effects of Caputo-Fabrizio fractional derivative for three different values $\alpha=$ $0.3,0.5,0.7$ on the profiles of the mass concentration. It is found that increasing Caputo-Fabrizio fractional derivative parameter increases the concentration profile. 


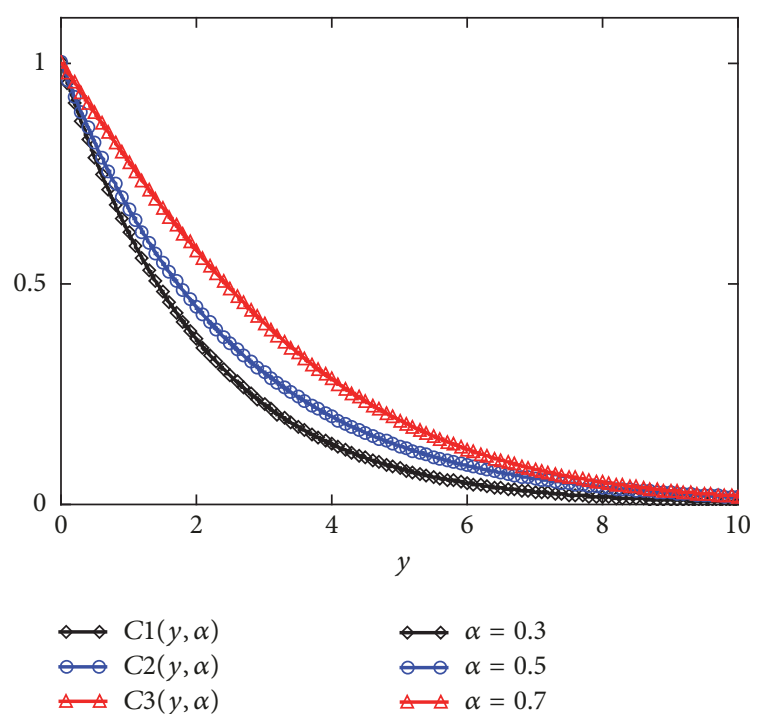

FIgUre 2: Plot of mass concentration for different values of CaputoFabrizio fractional parameter $\alpha$.

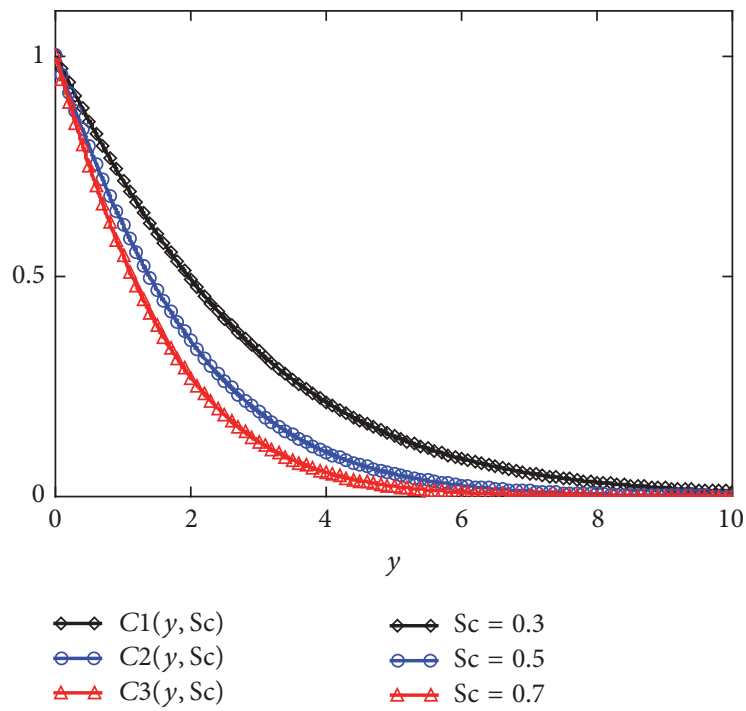

Figure 3: Plot of mass concentration for different values of Schmidt number Sc.

(ii) Influence of the Schmidt number on the mass concentration is observed in Figure 3. It is noted that the increase in Schmidt number causes decrease in mass concentration.

(iii) Figure 4 emphasizes the effect of Caputo-Fabrizio fractional derivative for three different values $\alpha=$ $0.3,0.5,0.7$ on the profiles of the temperature distribution. It is noted that increase in Caputo-Fabrizio fractional parameter increases the temperature distribution.

(iv) Figure 5 depicts the effect of Prandtl number for three different values $\operatorname{Pr}=10,12,14$ on the profiles of the temperature distribution. Increasing Prandtl number causes decrease in the temperature distribution which

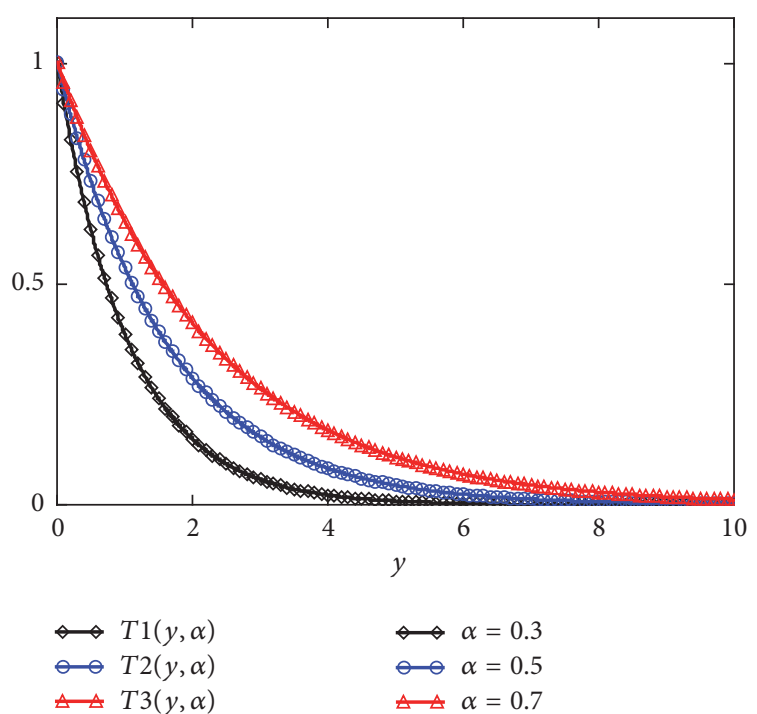

FIgURE 4: Plot of temperature distribution for different values of Caputo-Fabrizio fractional parameter $\alpha$.

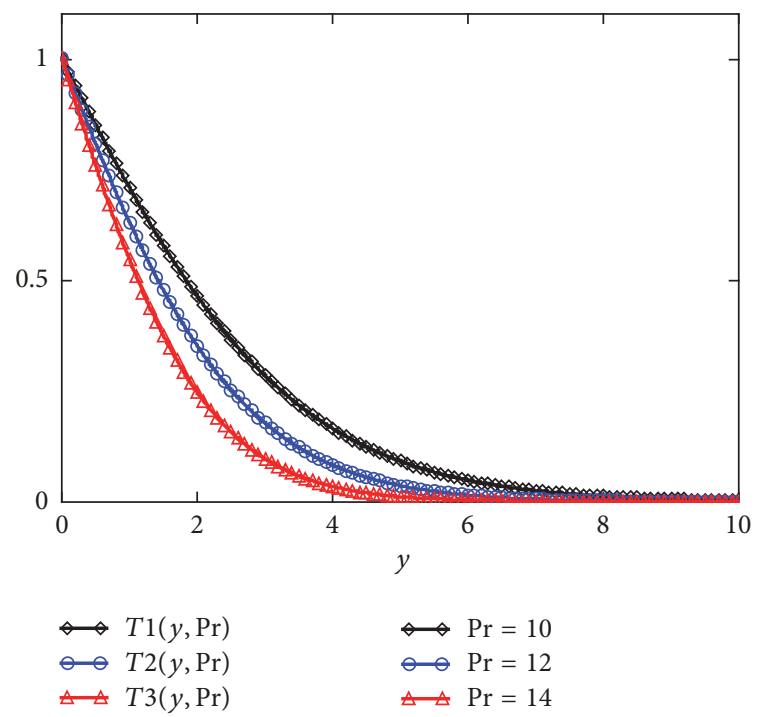

Figure 5: Plot of temperature distribution for different values of Prandtl number Pr.

is due to the fact that an increase in the Prandtl number generates slow rate of thermal diffusion over the whole domain of boundary.

(v) Figure 6 is portrayed to display the impacts of CaputoFabrizio fractional derivative $\alpha=0.2,0.4,0.6$ on velocity. It is found that velocity decreases with increasing values of fractional parameter.

(vi) The influence of Reynold number is depicted in Figure 7. It is noted that increasing values of Reynold numbers causes the velocity to decrease.

(vii) The influences of magnetic and porous parameters are demonstrated in Figures 8 and 9, respectively. As expected, flow of magnetohydrodynamic and porous 


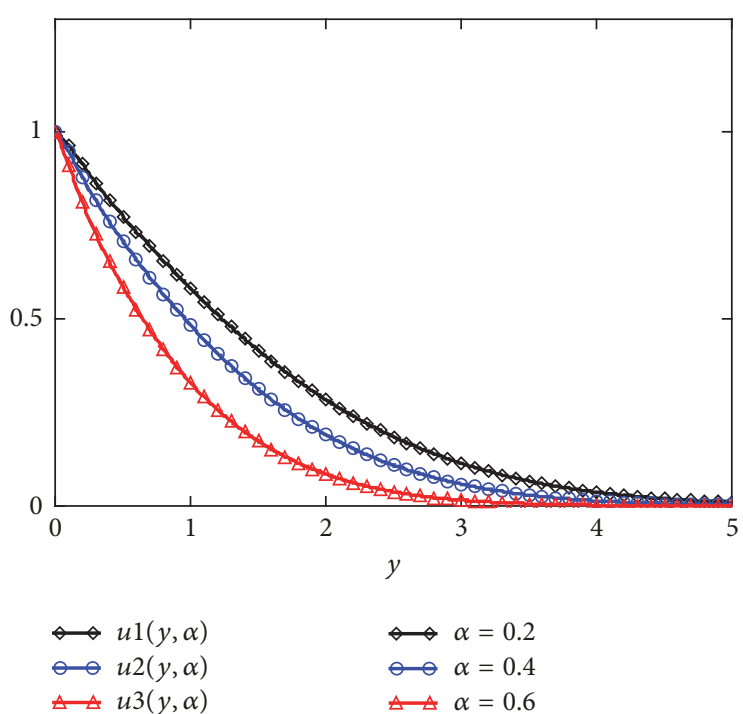

Figure 6: Plot of velocity field for different values of CaputoFabrizio fractional parameter $\alpha$.

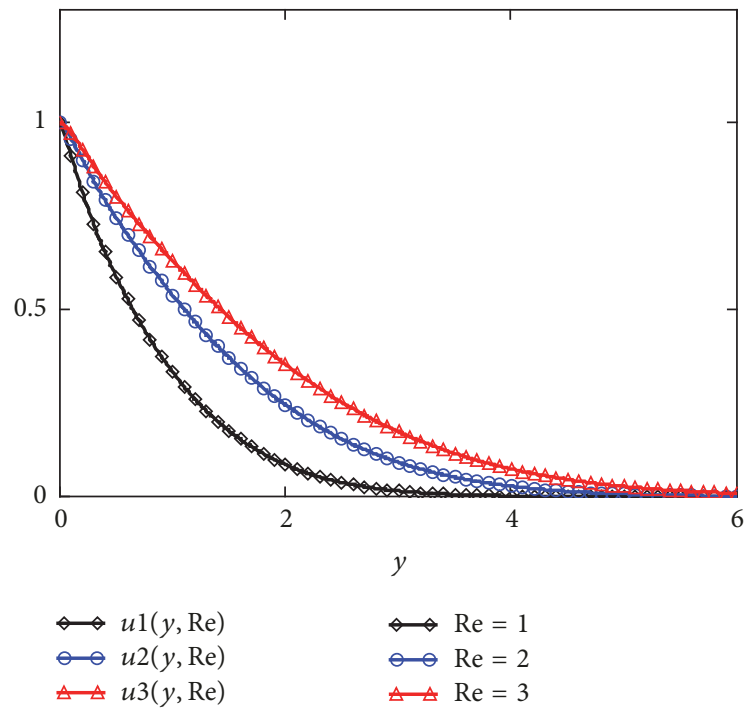

Figure 7: Plot of velocity field for different values of Reynold number Re.

flows have opposite effects on fluid on the whole domain of plate. It is also noted that both pertinent parameters have quite similar effects on fluid flow reciprocally.

\section{Conflicts of Interest}

The authors declare that there are no conflicts of interest regarding the publication of this paper.

\section{Authors' Contributions}

All authors contributed equally to the writing of this paper. All authors read and approved the final paper.

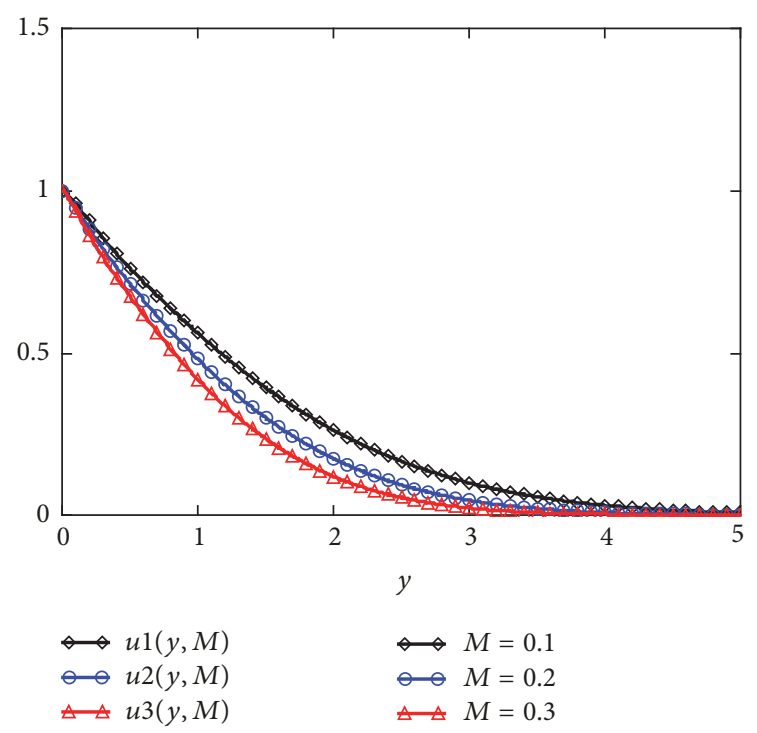

Figure 8: Plot of velocity field for different values of magnetic parameter $M$.

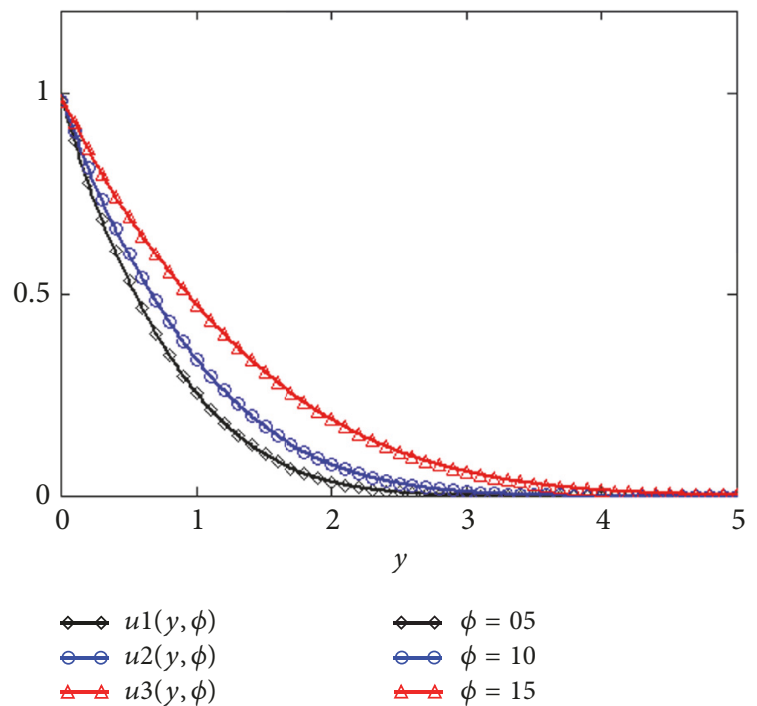

FIGURE 9: Plot of velocity field for different values of porous medium $\phi$.

\section{Acknowledgments}

The authors would like to acknowledge and express their gratitude to the United Arab Emirates University, Al Ain, UAE, for providing the financial support with Grant no. 31S240-UPAR (2) 2016.

\section{References}

[1] A. W. Sisko, "The flow of lubricating greases," Industrial \& Engineering Chemistry, vol. 50, no. 12, pp. 1789-1792, 1958.

[2] M. Qasim, "Heat and mass transfer in a Jeffrey fluid over a stretching sheet with heat source/sink," Alexandria Engineering Journal, vol. 52, no. 4, pp. 571-575, 2013. 
[3] I. Khan, K. Fakhar, and M. Anwar, "Hydromagnetic rotating flows of an oldroyd-B fluid in a porous medium," Special Topics and Reviews in Porous Media, vol. 3, no. 1, pp. 89-95, 2012.

[4] D. Tripathi and O. A. Beg, "Peristaltic propulsion of generalized Burgers' fluids through a non-uniform porous medium: A study of chyme dynamics through the diseased intestine," Mathematical Biosciences, vol. 248, pp. 67-77, 2014.

[5] K. A. Abro, A. A. Shaikh, and I. A. Junejo, "Analytical solutions under no slip effects for accelerated flows of Maxwell fluids," Sindh University Research Journal-SURJ, vol. 47, no. 3, pp. 613618, 2015.

[6] B. Sahoo and S. Poncet, "Flow and heat transfer of a third grade fluid past an exponentially stretching sheet with partial slip boundary condition," International Journal of Heat and Mass Transfer, vol. 54, no. 23-24, pp. 5010-5019, 2011.

[7] T. Sarpkaya and P. G. Rainey, "Stagnation point flow of a secondorder viscoelastic fluid," Acta Mechanica, vol. 11, no. 3-4, pp. 237-246, 1971.

[8] K. Walter, "Non-Newtonian effects in some elasticoviscous liquids whose behavior at small rates of shear is characterized by a general linear equation of state," The Quarterly Journal of Mechanics and Applied Mathematics, vol. 15, pp. 63-76, 1962.

[9] M. Caputo and M. Fabrizio, "A new definition of fractional derivative without singular kernel," Progress in Fractional Differentiation and Applications, vol. 1, no. 2, pp. 1-13, 2015.

[10] Q. M. Al-Mdallal, "On the numerical solution of fractional Sturm-Liouville problems," International Journal of Computer Mathematics, vol. 87, no. 12, pp. 2837-2845, 2010.

[11] A. A. Zafar and C. Fetecau, "Flow over an infinite plate of a viscous fluid with non-integer order derivative without singular kernel," Alexandria Engineering Journal, vol. 55, no. 3, pp. 27892796, 2016.

[12] S. G. Samko, A. A. Kilbas, and O. I. Marichev, "Fractional integrals and derivatives," in Theory and Applications, Gordon and Breach, Yverdon, Switzerland, 1993.

[13] Y. Y. Gambo, F. Jarad, D. Baleanu, and J. T. Abdel, "On Caputo modification of the Hadamard fractional derivatives," Advances in Difference Equations, vol. 1, pp. 1-10, 2014.

[14] T. Abdeljawad, F. Jarad, and D. Baleanu, "A semigroup-like property for discrete Mittag-Leffler functions," Advances in Difference Equations, vol. 1, pp. 72-84, 2012.

[15] T. Abdeljawad and D. Baleanu, "On fractional derivatives with exponential kernel and their discrete versions," Reports on Mathematical Physics, vol. 80, no. 1, pp. 11-27, 2017.

[16] A. Farhad, S. Muhammad, K. Ilyas, and A. S. Nadeem, "Application of Caputo-Fabrizio derivatives to MHD free convection flow of generalized Walters'-B fluid model," The European Physical Journal Plus, vol. 131, pp. 387-387, 2016.

[17] M. A. Hajji, Q. M. Al-Mdallal, and F. M. Allan, "An efficient algorithm for solving higher-order fractional Sturm-Liouville eigenvalue problems," Journal of Computational Physics, vol. 272, pp. 550-558, 2014.

[18] Q. M. Al-Mdallal and M. A. Hajji, "A convergent algorithm for solving higher-order nonlinear fractional boundary value problems," Fractional Calculus and Applied Analysis, vol. 18, no. 6, pp. 1423-1440, 2015.

[19] F. Ali, M. Saqib, I. Khan, and N. Ahmad Sheikh, "Application of Caputo-Fabrizio derivatives to MHD free convection flow of generalized Walters'-B fluid model," The European Physical Journal Plus, vol. 131, no. 10, pp. 377-389, 2016.

[20] A. Atangana and R. T. Alqahtani, "Numerical approximation of the space-time Caputo-Fabrizio fractional derivative and application to groundwater pollution equation," Advances in Difference Equations, vol. 1, pp. 156-161, 2016.

[21] B. S. T. Alkahtani and A. Atangana, "Analysis of non-homogeneous heat model with new trend of derivative with fractional order," Chaos, Solitons \& Fractals, vol. 89, pp. 566-571, 2016.

[22] J. Hristov, "Subdiffusion model with time-dependent diffusion coefficient: integral-balance solution and analysis," Thermal Science, vol. 21, pp. 69-80, 2017.

[23] A. Atangana and D. Baleanu, "New fractional derivatives with nonlocal and non-singular kernel: theory and application to heat transfer model," 2016, https://arxiv.org/abs/1602.03408.

[24] A. S. Nadeem, F. Ali, M. Saqib, I. Khan et al., "Comparison and analysis of the Atangana-Baleanu and Caputo-Fabrizio fractional derivatives for generalized Casson fluid model with heat generation and chemical reaction," Results in Physics, vol. 7, pp. 789-800, 2017.

[25] A. S. Nadeem, F. Ali, I. Khan, and M. Saqib, "A modern approach of Caputo-Fabrizio time-fractional derivative to MHD free convection flow of generalized second-grade fluid in a porous medium," Neural Computing and Applications, pp. 1-11, 2016.

[26] A. A. Kashif and A. S. Muhammad, "Heat transfer in magnetohydrodynamic second grade fluid with porous impacts using Caputo-Fabrizoi fractional derivatives," Punjab University Journal of Mathematics, vol. 49, no. 2, pp. 113-125, 2017.

[27] A. S. Nadeem, F. Ali, M. Saqib, I. Khan, and S. A. A. Jan, "A comparative study of Atangana-Baleanu and Caputo-Fabrizio fractional derivatives to the convective flow of a generalized Casson fluid," The European Physical Journal Plus, vol. 132, no. 1, article 54, 2017.

[28] J. Hristov, "Transient heat diffusion with a non-singular fading memory: from the cattaneo constitutive equation with Jeffrey's kernel to the caputo-fabrizio time-fractional derivative," Thermal Science, vol. 20, no. 2, pp. 757-762, 2016.

[29] A. A. Kashif, H. Mukarrum, and M. B. Mirza, "An analytic study of molybdenum disulfide nanofluids using modern approach of Atangana-Baleanu fractional derivatives," The European Physical Journal Plus, vol. 132, article 439, no. 10, 2017.

[30] A. Khan, K. A. Abro, A. Tassaddiq, and I. Khan, "AtanganaBaleanu and Caputo Fabrizio analysis of fractional derivatives for heat and mass transfer of second grade fluids over a vertical plate: a comparative study," Entropy, vol. 19, no. 8, pp. 1-12, 2017.

[31] A. Atangana and I. Koca, "Chaos in a simple nonlinear system with Atangana-Baleanu derivatives with fractional order," Chaos, Solitons \& Fractals, vol. 89, pp. 447-454, 2016.

[32] K. M. Owolabi and A. Atangana, "Numerical solution of fractional-in-space nonlinear Schrödinger equation with the Riesz fractional derivative," The European Physical Journal Plus, vol. 131, no. 9, article 335, 2016

[33] A. M. Mathai, R. K. Saxena, and H. J. Haubold, The H-Functions: Theory and Applications, Springer, New York, NY, USA, 2010.

[34] A. Atangana, "On the new fractional derivative and application to nonlinear Fisher's reaction-diffusion equation," Applied Mathematics and Computation, vol. 273, no. 1, pp. 948-956, 2016.

[35] H. L. Muzaffar, A. A. Kashif, and A. S. Asif, "Helical flows of fractional viscoelastic fluid in a circular pipe," International 
Journal of Advanced and Applied Sciences, vol. 4, no. 10, pp. 97105, 2017.

[36] M. Khan, T. Hayat, and S. Asghar, "Exact solution for MHD flow of a generalized Oldroyd-B fluid with modified Darcy's law," International Journal of Engineering Science, vol. 44, no. 5-6, pp. 333-339, 2006. 


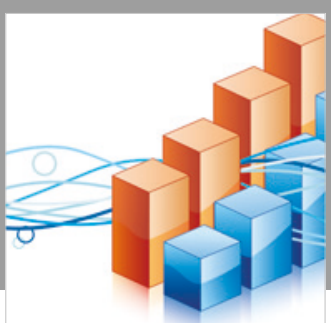

Advances in

Operations Research

\section{-n-m}
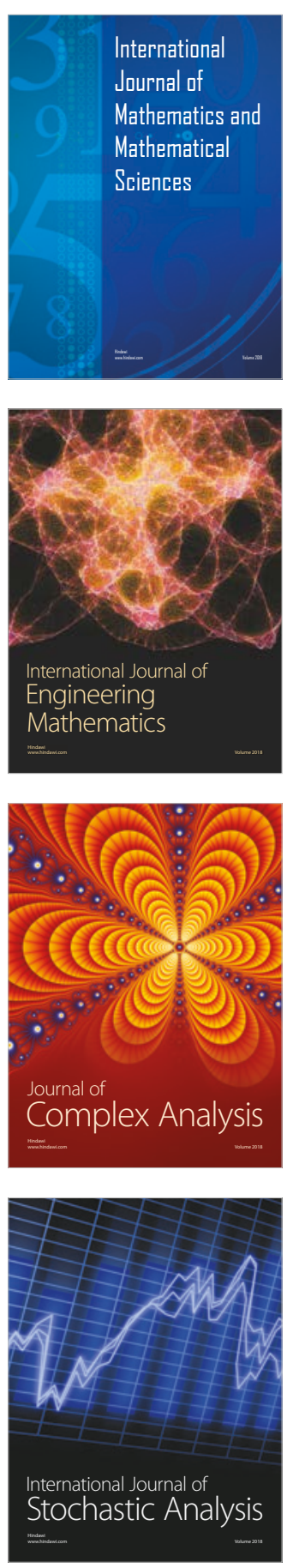
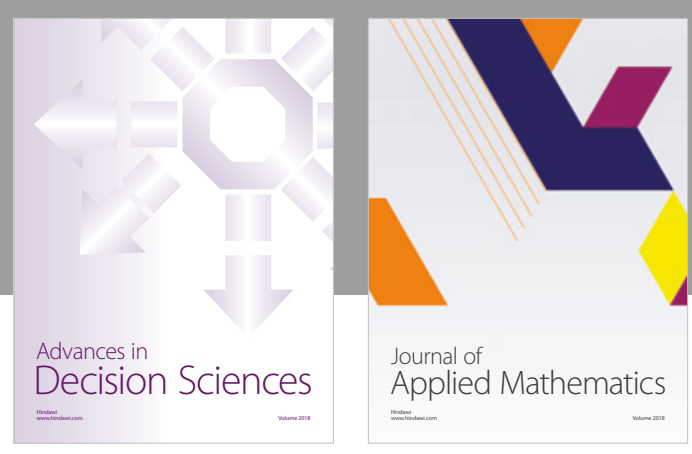

Journal of

Applied Mathematics
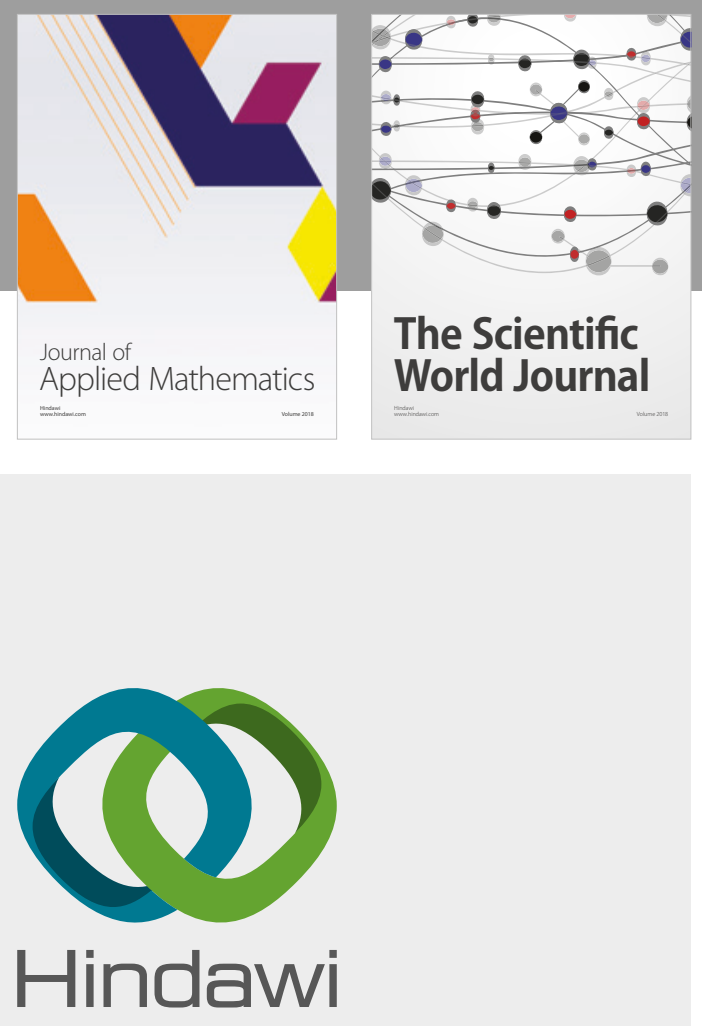

Submit your manuscripts at

www.hindawi.com

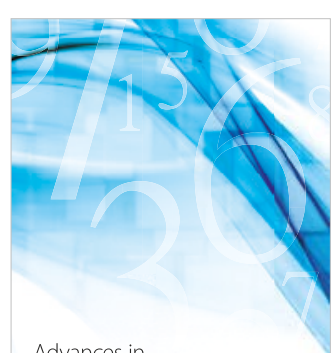

Advances in
Numerical Analysis
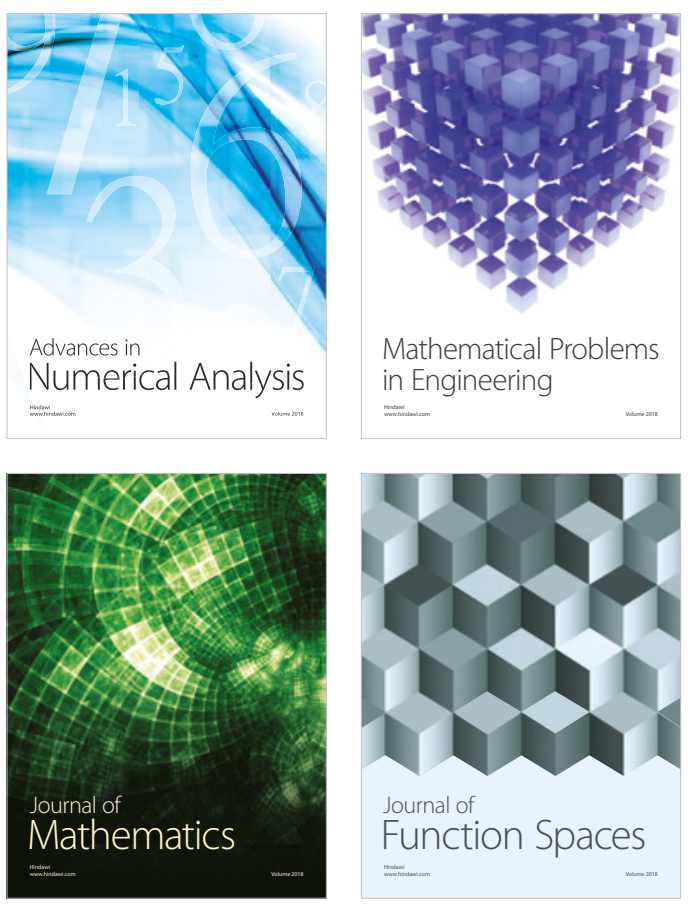

Mathematical Problems in Engineering

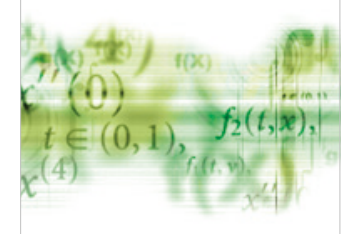

International Journal of

Differential Equations

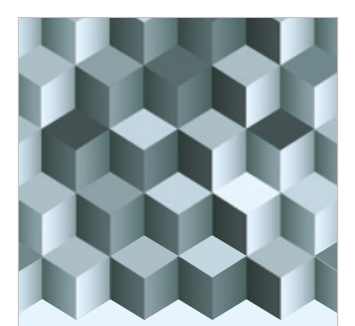

Journal of

Function Spaces
The Scientific

World Journal

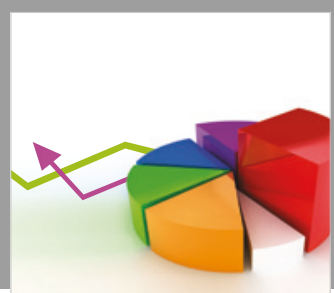

Journal of

Probability and Statistics
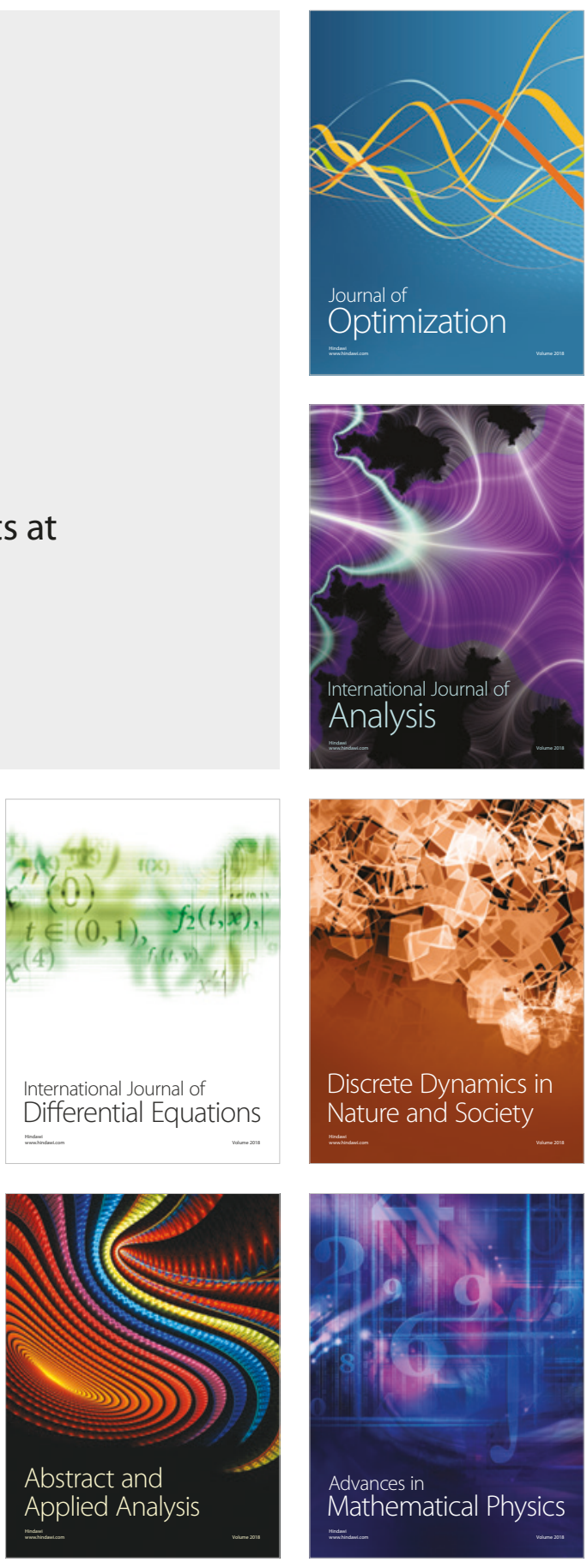